\title{
Clinical potential of necitumumab in non-small cell lung carcinoma
}

This article was published in the following Dove Press journal:

OncoTargets and Therapy

31 August 2016

Number of times this article has been viewed

\author{
Carlo Genova ${ }^{1-3}$ \\ Fred R Hirsch' \\ 'Division of Medical Oncology, \\ Department of Medicine, University \\ of Colorado Cancer Center, Aurora, \\ CO, USA; ${ }^{2}$ Lung Cancer Unit, IRCCS \\ AOU San Martino IST, ${ }^{3}$ Department of \\ Internal Medicine, School of Medicine, \\ University of Genoa, Genoa, Italy
}

\begin{abstract}
Despite significant progress, new therapeutic approaches for advanced non-small cell lung cancer (NSCLC) are highly needed, particularly for the treatment of patients with squamous cell carcinoma. The epidermal growth factor receptor (EGFR) is often overexpressed in NSCLC and represents a relevant target for specific treatments. Although EGFR mutations are more frequent in non-squamous histology, the receptor itself is more often overexpressed in squamous NSCLC. Necitumumab is a human monoclonal antibody that is able to inhibit the EGFR pathway and cause antibody-dependent cell cytotoxicity. This drug has been studied in combination with first-line chemotherapy for advanced NSCLC in two Phase III trials, and a significant survival benefit was reported in squamous NSCLC (SQUIRE trial); by contrast, necitumumab did not prove itself beneficial in non-squamous histotype (INSPIRE trial). On the basis of the SQUIRE results, necitumumab was approved in combination with cisplatin and gemcitabine as a first-line treatment for advanced squamous NSCLC, both in the US and Europe, where its availability is limited to patients with EGFRexpressing tumors. The aim of this review is to describe the tolerability and the efficacy of necitumumab by searching the available published data and define its potential role in the current landscape of NSCLC treatment.
\end{abstract}

Keywords: necitumumab, EGFR, non-small cell lung cancer, monoclonal antibody, H-score

\section{Introduction}

Non-small cell lung cancer (NSCLC) accounts for a significant proportion of the global cancer-related deaths in spite of continuous therapeutic improvements. Most patients are diagnosed with advanced disease and, because curative treatments are not available at this stage, the only available option is palliative systemic therapy, which aims at prolonging survival and improving symptoms. ${ }^{1}$ Different histologic subtypes of NSCLC can be identified, the most representative being adenocarcinoma (ADC) and squamous cell carcinoma (SCC). Currently, therapeutic options for SCC are more limited compared to ADC, as the latter subtype can be treated with "new" agents like pemetrexed and bevacizumab, as well as specific targeted therapies when sensitizing mutations of the epidermal growth factor receptor $(E G F R)$ gene or rearrangements involving the anaplastic lymphoma kinase $(A L K)$ gene are identified. ${ }^{2}$

Notably, although activating mutations of the EGFR gene are more typical of non-squamous histology, the EGFR protein itself is often highly expressed in both histologic subtypes, and it has been reported that its over-expression in SCC is even more pronounced than in ADC ( $82 \%$ vs $44 \%){ }^{3}$ hence, it has been postulated that EGFR could represent an eligible target for both histotypes. Cetuximab, a chimeric murine/human monoclonal antibody $(\mathrm{mAB})$ targeting EGFR, currently registered for
Correspondence: Fred R Hirsch Department of Medicine, Division of Medical Oncology, University of Colorado Cancer Center, I280I E. 17th Avenue, 80045 Aurora, CO, USA Tel + I 3038079853

Email fred.hirsch@ucdenver.edu 
the treatment of colorectal and head and neck cancers, was studied in combination with first-line chemotherapy in an open-label, Phase III trial involving patients with advanced NSCLC who were not selected for histology (FLEX trial), achieving a significant advantage in terms of overall survival (OS) compared to chemotherapy alone (11.3 vs 10.1 months; hazard ratio $[\mathrm{HR}]=0.871 ; P=0.044) ;{ }^{4}$ a retrospective analysis suggested that the OS advantage achieved with the addition of cetuximab to chemotherapy was limited to patients with high expression of EGFR, while this benefit was not confirmed in patients with low expression of the protein. ${ }^{5}$

Necitumumab (IMC-11F8, LY3012211) is a secondgeneration human $\mathrm{mAB}$ directed against the extracellular region of EGFR, developed by Eli Lilly \& Co (Indianapolis, IN, USA); other agents in the same drug class include cetuximab and panitumumab, which are available for clinical use in other solid malignancies. ${ }^{6}$

Recently, significant data regarding activity, efficacy, and tolerability of necitumumab in NSCLC have been collected and the aim of this review is to define the potential role of this drug in the treatment of NSCLC by exploring the available literature; for this purpose, relevant articles indexed in PubMed and abstracts from major oncology conferences have been considered.

\section{Pharmacology Pharmacodynamics}

Under normal conditions, when EGFR interacts with its ligand, the epidermal growth factor, the former undergoes dimerization and subsequent phosphorylation, leading to the activation of downstream signaling pathways that involve a number of subsequent protein-based cascades, such as the Kirsten rat sarcoma/rapidly accelerated fibrosarcoma/mitogen-activated protein kinase pathway and the phosphatidylinositol-3-kinase/protein kinase B pathway; these signaling cascades ultimately lead to the promotion of cell proliferation and survival. While this process has a physiologic role in the development of tissues and organs, it is also a key player in the growth of several types of neoplasms, including lung cancer. ${ }^{7}$ Necitumumab contains an antigen-binding fragment, known as FAB-11F8, with high affinity for one of the ligand-binding extracellular domains of EGFR; ${ }^{8}$ as a consequence of competitive binding, the mAB prevents the dimerization of EGFR and the subsequent signaling cascade, resulting in internalization and degradation of the receptor, ultimately followed by inhibition of proliferation and survival. ${ }^{9}$ Additionally, since necitumumab belongs to the immunoglobulin (Ig) G1 class, it retains the ability to induce antibody-dependent cell cytotoxicity (ADCC) by stimulating response from adaptive immune cells (such as lymphocytes), which exert their activity on opsonized neoplastic cells (eg, activating the perforin/ granzymes mechanism), resulting in immune-mediated cell lysis. ${ }^{10,11}$ The mechanisms of action of necitumumab are summarized in Figure 1.

\section{Pharmacokinetics}

Necitumumab shares common pharmacokinetic features with other therapeutic mABs, which are unavailable by oral route and get distributed in tissues at slow speed and low volumes, resulting in increased half-life compared to non-antibody drugs; additionally, the elimination routes of such agents are only partially documented, and involve degradation to peptides after being internalized into their target cells, proteolysis within the liver or the reticuloendothelial system, as well as nonspecific endocytosis. In a first-in-human, dose-escalation, Phase I trial, the maximum tolerated dose of necitumumab was identified to be $800 \mathrm{mg}$; at this dose, the half-life of necitumumab was $\approx 7$ days; additionally, its mean clearance decreased in a less than dose-proportional pattern, while its maximum serum concentration and area under the concentration versus time curve extrapolated from time 0 to infinity values increased disproportionately to the dose of necitumumab. These nonlinear pharmacokinetics findings suggest a saturable clearance mechanism for this drug; additionally, its metabolism is not apparently related to height or weight, indicating fixed dose as the most suitable choice for necitumumab. ${ }^{12,13}$

\section{Preclinical data}

Necitumumab was able to inhibit in vitro proliferation of a series of tumor cell lines characterized by overexpression of EGFR, including epidermal, pancreatic, and colorectal cell lines. ${ }^{14}$ Additionally, the binding and ADCC activities of necitumumab were subjects of comparisons with cetuximab and panitumumab; in colorectal cells, necitumumab showed an EGFR binding activity similar to cetuximab and significantly higher than panitumumab, although panitumumab had a higher affinity for the receptor, ${ }^{15}$ in NSCLC cells, necitumumab and cetuximab, both belonging to the IgG1 class, were rapidly internalized into target cells and were able to induce a relevant ADCC at $1.0 \mathrm{nM}$ with a magnitude directly proportional to EGFR expression, while panitumumab was internalized more slowly and, being an IgG2, was not able to activate ADCC. ${ }^{16,17}$ Finally, since cetuximab was associated with hypersensitivity reactions in clinical practice, 


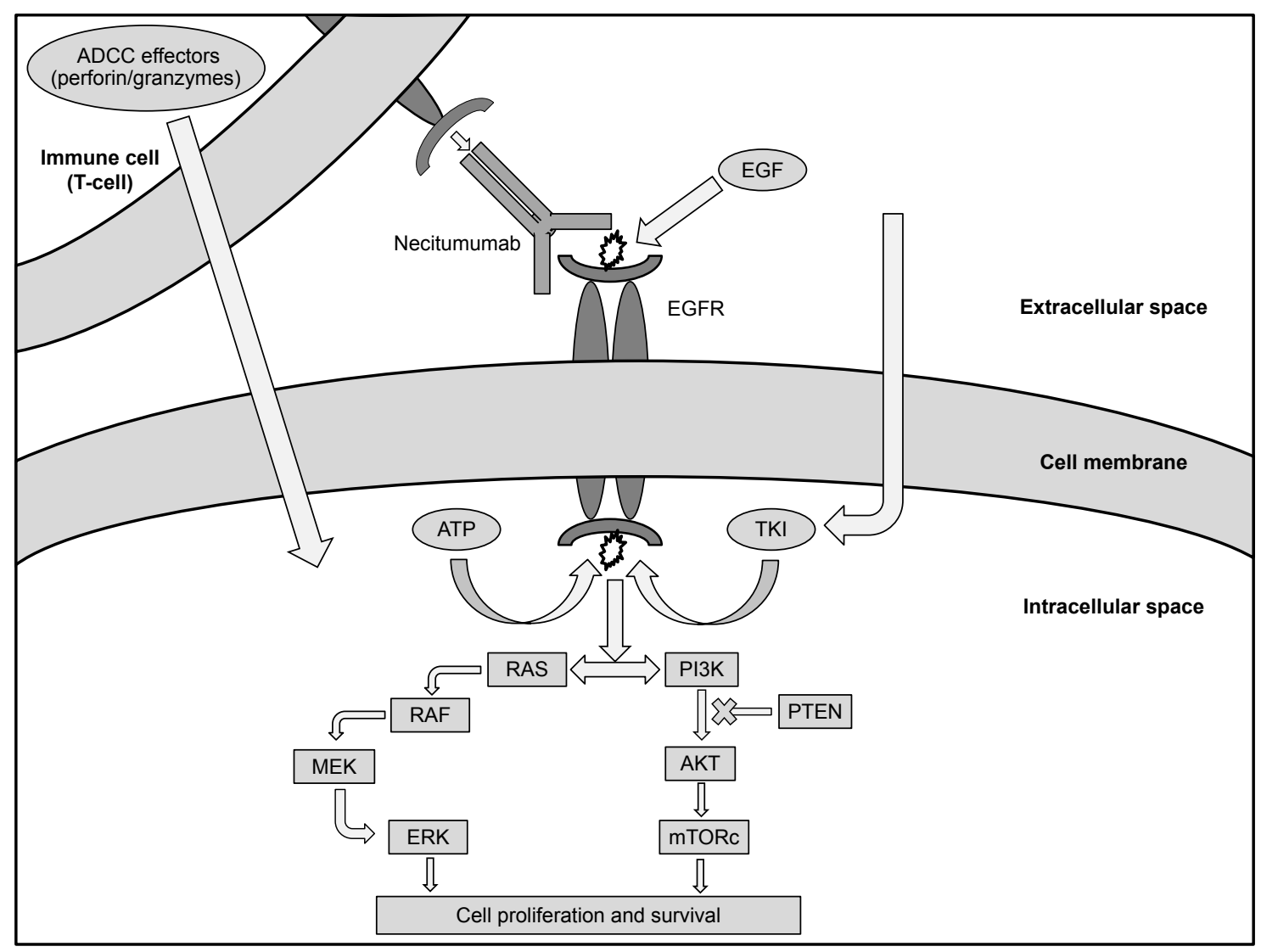

Figure I In normal conditions, the extracellular interaction between EGFR and its ligand, EGF, leads to dimerization of the receptor, binding with ATP in the intracellular region of the receptor, and activation of two main pathways, the first being RAS-RAF-MEK-ERK and the second being PI3K-AKT-mTORc (which is inhibited by PTEN). Notes: Both pathways ultimately lead to proliferative and anti-apoptotic signals. In tumor cells with overexpressed or mutated EGFR, this mechanism is generally overactivated. Necitumumab binds the extracellular region of EGFR, preventing its interaction with EGF and the following downstream cascade. Additionally, the necitumumab-EGFR complex can induce ADCC by various types of immune system cells, such as T lymphocytes, NK lymphocytes, and macrophages; in the example, an activated cytotoxic T-cell releases ADCC effectors (the perforin/granzyme system), ultimately leading to the death of the target cell. The figure shows also the different mechanism of action of EGFR-TKIs, which inhibit the binding with ATP in the intracellular space and consequent activation of the aforementioned cascade.

Abbreviations: ADCC, antibody-dependent cell cytolysis; AKT, protein kinase B; ATP, adenosine triphosphate; EGF, epidermal growth factor; EGFR, epidermal growth factor receptor; ERK, extracellular signal-regulated kinase; MEK, mitogen-activated protein kinase kinase; mTORc, mammalian target of rapamycin complex; NK, natural killer; PI3K, phosphatidylinositol-3-kinase; PTEN, phosphatase and tensin homolog; RAF, rapidly accelerated fibrosarcoma; RAS, rat sarcoma; TKI, tyrosine kinase inhibitor.

potentially related to its chimeric structure (mouse/human), the allergenic potentials of cetuximab, necitumumab, and panitumumab were evaluated, measuring the activity of anti-galactose-alpha-1,3-galactose IgE as a proper marker: notably, both necitumumab and panitumumab, which are fully-human mABs, induced a significantly lower IgE activity compared to cetuximab. ${ }^{18}$

Necitumumab confirmed its tumor inhibiting activity, alone or in combination with chemotherapy, also against epidermoid, colorectal, and pancreatic human xenograft models in immunosuppressed mice. ${ }^{19}$ Notably, among several antineoplastic treatments employed on NSCLC xenograft models, including necitumumab in combination with pemetrexed, paclitaxel, or cisplatin-gemcitabine doublet, the latter combination resulted particularly effective in terms of tumor shrinkage; further data suggest that part of the activity of this combination might be connected with the overexpression of a microRNA, hsa-miR-29b, and the down-regulation of DNA (cytosine-5)-methyltransferase $3 \mathrm{~B} .^{20}$

\section{Clinical trials}

\section{Phase I trials}

The first-in-human, dose-escalation, Phase I trial involving necitumumab included 60 patients affected by advanced solid tumors, including colo-rectal, esophageal, ovarian, pancreatic, prostate, renal, gastric, and lung cancer, who were randomized to a weekly schedule (arm A; 29 patients) or to an every 2-week schedule (arm B; 31 patients); each arm included six escalating cohorts of three to four patients receiving different doses of the drug (100-200-400-600-800-1,000 mg). Each patient received a single administration of necitumumab at the planned dose, followed by 2 weeks of "rest" from 
treatment for collection of pharmacokinetic data (already described in the "pharmacokinetics" paragraph), followed by subsequent administrations according to the treatment arm and cohort. Necitumumab was globally well tolerated in both arms, its most common toxicity being acneiform rash, generally mild (all grades: $55.2 \%$; grade $\geq 3 \%$ : $3.3 \%$ ), grade $1-2$ diarrhea (41.4\% in arm $\mathrm{A}$ and $19.4 \%$ in arm B; no grade $\geq 3$ reported), and grade $1-2$ nausea (10.3\% in arm $A$ and $22.6 \%$ in arm $B$; no grade $\geq 3$ reported). Two patients from arm B enrolled in the 1,000 mg cohort experienced grade 3 headache associated with nausea, vomiting, and fever; this event was considered a dose-limiting toxicity and consequently the maximum tolerated dose was set at $800 \mathrm{mg}$. Globally, 47 patients were evaluable for response: disease stabilizations in 16 (eight in each arm) and partial responses in two (one in each arm) were reported; 17 patients achieved a progression-free survival (PFS) of 3 months and nine reached a PFS of 6 months. ${ }^{13}$

Based on these findings, another Phase I trial was started in order to evaluate the pharmacologic characteristics of necitumumab in a population of Asian patients; in this study, 15 Japanese patients received necitumumab in three different cohorts: $600 \mathrm{mg}$ on days 1 and 8 every 3 weeks, $800 \mathrm{mg}$ on day 1 every 3 weeks, and $800 \mathrm{mg}$ on days 1 and 8 every 3 weeks (no escalation over $800 \mathrm{mg}$ was planned, as this was the maximum tolerated dose in the previous trial). In this trial, no dose-limiting toxicity was reported at 600 or $800 \mathrm{mg}$, while the most frequent adverse events were headache (66.7\%), dry skin $(66.7 \%)$, pruritus $(53.3 \%)$, and skin rash $(53.3 \%)$, and most of them were mild or moderate (grade 1-2); a disease control rate (DCR) of $67 \%$ was reported. ${ }^{21}$

\section{Phase II trials}

Phase II trials involving necitumumab in NSCLC are relatively limited, and most available data have been collected from conference abstracts. In a single-arm Phase II trial, necitumumab (800 $\mathrm{mg}$ on days 1 and 8 ) was administered in combination with cisplatin $\left(75 \mathrm{mg} / \mathrm{m}^{2}\right.$ on day 1$)$ and gemcitabine $\left(1,250 \mathrm{mg} / \mathrm{m}^{2}\right.$ on days 1,8$)$ in a maximum of six 3 -week cycles as first-line treatment for 61 patients affected by advanced squamous NSCLC with the possibility to continue necitumumab as maintenance in case of responding or stable disease. Response rate (RR) and DCR were $48.1 \%$ and 81.5\%, while median PFS and OS were 5.6 and 11.7 months, respectively. ${ }^{22}$

In another Phase II trial, 167 patients with stage IV squamous NSCLC were randomized (2:1) to receive either carboplatin (area under the curve $=6$ on day 1 ), paclitaxel
(200 $\mathrm{mg} / \mathrm{m}^{2}$ on day 1 ), and necitumumab (800 $\mathrm{mg}$ on days 1 and 8 ) or only carboplatin and paclitaxel every 21 days for a maximum of six cycles; the patients enrolled in the experimental arm could continue necitumumab as monotherapy in case of response or stabilization after completion of the combination treatment. Globally, RR was $48.9 \%$ in the experimental arm and $40.0 \%$ in the control arm, while DCR was $87.2 \%$ and $84.0 \%$, respectively; the addition of necitumumab, compared to chemotherapy alone, did not result in significant differences in terms of median OS (13.2 vs 11.2 months; HR=0.83; $P=0.379$ ) or PFS (5.4 vs 5.6 months; $\mathrm{HR}=1.0 ; P$ not reported). Grade 3 hypomagnesemia and rash were more frequent in the experimental arm $(5.7 \%$ vs $0 \%$ and $2.8 \%$ vs $0 \%$, respectively), while thromboembolic events of any grade were similar $(3.8 \%$ vs $3.6 \%){ }^{23}$

\section{Phase III trials}

The clinical efficacy of necitumumab combination with first-line chemotherapy for advanced NSCLC was evaluated in two large randomized, Phase III trials; one addressing patients with non-squamous histology (INSPIRE) ${ }^{24}$ and one addressing patients with squamous histology (SQUIRE). ${ }^{25}$ The efficacy data of these trials have been summarized in Table 1. Both studies included a preplanned analysis of the EGFR expression, which was defined in the form of an immunohistochemistry (IHC) value called H-score, ${ }^{3}$ which was also employed in the retrospective analysis of the FLEX trial with cetuximab ${ }^{5}$ and was computed on the basis of the percentage of cells expressing each IHC value (ranging from 0 to $3+)$ with the following formula: $(1 \times \%$ of cells expressing IHC $1+)+(2 \times \%$ of cells expressing IHC $2+)+(3 \times \%$ of cells expressing IHC $3+)$, resulting in a value between 0 and 300. In the INSPIRE study (non-squamous carcinomas), the expression of EGFR was considered low if $\mathrm{H}$-score was $<200$, and high if $\mathrm{H}$-score was $\geq 200$; this cutoff score was derived from the post hoc analysis of FLEX, where the addition of cetuximab to chemotherapy achieved a significant OS advantage in patients belonging to the high $\mathrm{H}$-score population, while patients with low $\mathrm{H}$-score had no advantage. The outcome data of the INSPIRE and SQUIRE trials based on H-score are reported in Table 2.

In the INSPIRE trial, patients with previously untreated, stage IV, non-squamous NSCLC were randomized (1:1) to receive 3-week cycles of treatment with cisplatin $75 \mathrm{mg} / \mathrm{m}^{2}$ plus pemetrexed $500 \mathrm{mg} / \mathrm{m}^{2}$ on day 1 , either alone or in combination with necitumumab $800 \mathrm{mg}$ on days 1 and 8 for a maximum of six cycles, with the option of receiving necitumumab monotherapy at the same dose for the patients in the experimental 
Table I Trial design and efficacy results of the INSPIRE and the SQUIRE trials

\begin{tabular}{|c|c|c|}
\hline Trial & INSPIRE ${ }^{24}$ & SQUIRE 25 \\
\hline Trial design & Open-label, randomized Phase III trial & Open-label, randomized Phase III trial \\
\hline Addressed population & Treatment-naïve, stage IV nonsquamous NSCLC & Treatment-naïve, stage IV squamous NSCLC \\
\hline Treatment arms & $\begin{array}{l}\text { Exp: Cisplatin }\left(75 \mathrm{mg} / \mathrm{m}^{2} \text { day I) + pemetrexed }\right. \\
\left(500 \mathrm{mg} / \mathrm{m}^{2} \text { day I) + necitumumab }(800 \mathrm{mg} \text { days I and } 8)\right. \\
\text { every } 3 \text { weeks for six cycles followed by maintenance with } \\
\text { necitumumab ( } 800 \mathrm{mg} / \mathrm{m}^{2} \text { days I and } 8 \text { ) every } 3 \text { weeks after } \\
\text { the sixth cycle (until death, PD, or unacceptable toxicity) } \\
\text { Ctrl: Cisplatin ( } 75 \mathrm{mg} / \mathrm{m}^{2} \text { day I) + pemetrexed } \\
\left(500 \mathrm{mg} / \mathrm{m}^{2} \text { day I) every } 3 \text { weeks for six cycles }\right.\end{array}$ & $\begin{array}{l}\text { Exp: Cisplatin }\left(75 \mathrm{mg} / \mathrm{m}^{2} \text { day I) + gemcitabine }\right. \\
\left(\mathrm{I}, 250 \mathrm{mg} / \mathrm{m}^{2} \text { days I and } 8\right)+\text { necitumumab }(800 \mathrm{mg} \\
\text { days I and } 8 \text { ) every } 3 \text { weeks for six cycles followed } \\
\text { by maintenance with necitumumab }\left(800 \mathrm{mg} / \mathrm{m}^{2} \text { days I }\right. \\
\text { and } 8) \text { every } 3 \text { weeks after the sixth cycle (until death, } \\
\text { PD, or unacceptable toxicity) } \\
\text { Ctrl: Cisplatin }\left(75 \mathrm{mg} / \mathrm{m}^{2} \text { day I) + gemcitabine }\right. \\
\left(\mathrm{I}, 250 \mathrm{mg} / \mathrm{m}^{2} \text { days I and } 8 \text { ) every } 3 \text { weeks for six cycles }\right.\end{array}$ \\
\hline Randomization ratio & $\mathrm{I}: \mathrm{I}$ & $\mathrm{I}: \mathrm{I}$ \\
\hline $\begin{array}{l}\text { Number of evaluable } \\
\text { patients }\end{array}$ & $633(315$ Exp and $318 \mathrm{Ctrl})$ & I,093 (545 Exp and $548 \mathrm{Ctrl})$ \\
\hline \multirow[t]{2}{*}{ Endpoints } & Primary: OS & Primary: OS \\
\hline & Secondary: DCR, ORR, PFS, PRO, TTF & Secondary: DCR, ORR, PFS, PRO, TTF \\
\hline Median OS (Exp vs Ctrl) & II.3 vs II.5 months $(\mathrm{HR}=\mathrm{I} .0 \mathrm{I} ; 95 \% \mathrm{Cl}=0.84-\mathrm{I} .2 \mathrm{I} ; \mathrm{P}=0.96)$ & II.5 vs 9.9 months $(\mathrm{HR}=0.84 ; 95 \% \mathrm{Cl}=0.74-0.96 ; P=0.0 \mathrm{I})$ \\
\hline Median PFS (Exp vs Ctrl) & 5.6 vs 5.6 months $(\mathrm{HR}=0.96 ; 95 \% \mathrm{Cl}=0.80-\mathrm{I} .16 ; P=0.66)$ & 5.7 vs 5.5 months $(\mathrm{HR}=0.85 ; 95 \% \mathrm{Cl}=0.74-0.98 ; P=0.02)$ \\
\hline Median TTF (Exp vs Ctrl) & 3.5 vs 4.3 months $(H R=I .18 ; P=0.046)$ & 4.3 vs 3.6 months $(H R=0.84 ; P=0.006)$ \\
\hline ORR (Exp vs Ctrl) & $31 \%$ vs $32 \%(P=0.79)$ & $31 \%$ vs $28 \%(P=0.40)$ \\
\hline DCR (Exp vs Ctrl) & $73 \%$ vs $74 \%$ ( $P$ not reported $)$ & $82 \%$ vs $77 \%(P=0.043)$ \\
\hline ClinicalTrials.gov & NCT00982 I II & NCT00981058 \\
\hline
\end{tabular}

Abbreviations: $95 \% \mathrm{Cl}$, 95\% confidence interval; CTCAE, common terminology criteria for adverse events (version 3.0); Ctrl, control arm; DCR, disease control rate; Exp, experimental arm; HR, hazard ratio; NSCLC, non-small cell lung cancer; ORR, objective response rate; OS, overall survival; PD, progressive disease; PFS, progression-free survival; PRO, patient reported outcome; TTF, time to treatment failure.

arm who remained stable or responding after the completion of combination treatment; the trial was designed as open label, because the known necitumumab-related skin toxicity would have prevented an effective blinding. After 15 months, an imbalance in deaths due to any cause and in grade 5 thromboembolic events was observed and hence the independent data monitoring committee recommended stopping enrollment and determined that, as a consequence of such events, the study was highly unlikely to achieve a survival benefit. ${ }^{26}$ At that time, 633 patients had been randomized and data from 616 patients (304 in the experimental arm and 312 in the control arm) were available for the planned outcome analyses. The authors stated that severe (grade $\geq 3$ ) adverse events were more frequent in the arm containing necitumumab than in the arm containing chemotherapy alone ( $51 \%$ vs $41 \%$ ), including possibly specific treatment-related fatal adverse events ( $5 \%$ vs $3 \%$ ).

In the experimental arm, the incidence of venous thromboembolism of any grade was $13 \%$ while that of grades 3,4 , and 5 were $6 \%,<1 \%$, and $1 \%$, respectively; in the control arm, incidence of any grade was $8 \%$, while that of grades 3,4 , and 5 were $2 \%,<1 \%$, and $1 \%$, respectively. Skin toxicity was the most common adverse event observed with necitumumab (any grade: $78 \%$, grade $\geq 3 \%$ : 16\%) in comparison with the control arm (any grade: 19\%, grade $\geq 3 \%$ : $<1 \%$ ). The efficacy analysis showed that adding necitumumab to chemotherapy did not result in improvements in terms of PFS (5.6 vs 5.6 months; HR $=0.96$; 95\% confidence intervals $(\mathrm{CI})=0.90-1.16 ; P=0.66)$ or $\mathrm{OS}(11.3$ vs 11.5 months; $\mathrm{HR}=1.01 ; 95 \% \mathrm{CI}=0.84-1.21 ; P=0.96)$; additionally, overall RR was not different between the experimental and the control arm $(31 \%$ vs $32 \%$; odds ratio $(\mathrm{OR})=0.96 ; 95 \%$ $\mathrm{CI}=0.68-1.34 ; P=0.79)$. Globally, 490 patients were evaluable for H-score, of which 290 (59\%) were H-score low, while 200 (41\%) were $\mathrm{H}$-score high; H-score was unable to predict improved outcomes with the combination, including necitumumab: in the $\mathrm{H}$-score low patients, the experimental and the standard arm were not different in terms of PFS ( 4.9 vs 4.8 months; HR $=0.95 ; 95 \% \mathrm{CI}=0.73-1.23 ; P=0.68)$, OS (9.0 vs 9.7 months; $\mathrm{HR}=1.07 ; 95 \% \mathrm{CI}=0.83-1.38 ; P=0.59$ ), and RR (27\% vs $26 \%$; OR=1.06; $95 \% \mathrm{CI}=0.63-1.78$; $P=0.84)$; similarly, among the $\mathrm{H}$-score high patients, no difference was observed between the experimental and the standard arm in terms of PFS (5.6 vs 5.6 months; HR $=0.94$; $95 \% \mathrm{CI}=0.68-1.30 ; P=0.71)$, OS (15.0 vs 13.3 months; $\mathrm{HR}=1.03 ; 95 \% \mathrm{CI}=0.75-1.43 ; P=0.85)$, and $\mathrm{RR}(40 \%$ vs $39 \%$; OR $=1.01 ; 95 \% \mathrm{CI}=0.57-1.78 ; P=0.98){ }^{24}$

In the other Phase III trial, SQUIRE, 1,093 patients with advanced SCC were randomized $(1: 1)$ to receive firstline chemotherapy with cisplatin $75 \mathrm{mg} / \mathrm{m}^{2}$ on day 1 plus gemcitabine $1,250 \mathrm{mg} / \mathrm{m}^{2}$ on days 1 and 8 administered 
Table 2 Outcome data for subpopulations based on EGFR H-score in the INSPIRE and SQUIRE trials

\begin{tabular}{|c|c|c|c|c|c|c|c|}
\hline & \multicolumn{7}{|l|}{ INSPIRE $^{24}$} \\
\hline & \multicolumn{3}{|l|}{ H-score high } & & \multicolumn{3}{|l|}{ H-score low } \\
\hline & \multicolumn{2}{|l|}{ CDDP-PEM-NEC } & \multicolumn{2}{|l|}{ CDDP-PEM } & \multicolumn{2}{|l|}{ CDDP-PEM-NEC } & \multirow{2}{*}{$\begin{array}{l}\text { CDDP-PEM } \\
146\end{array}$} \\
\hline Patients, $\mathrm{n}$ & 101 & & 99 & & 144 & & \\
\hline \multicolumn{8}{|l|}{ Overall survival } \\
\hline Median (months) & 15.0 & & 13.3 & & 9.0 & & 9.7 \\
\hline Hazard ratio & 1.03 & & & & 1.07 & & \\
\hline$P$-value & & 0.85 & & & & 0.59 & \\
\hline Interaction $P$-value & & & & 0.86 & & & \\
\hline \multicolumn{8}{|c|}{ Progression-free survival } \\
\hline Median (months) & 5.6 & & 5.6 & & 4.9 & & 4.8 \\
\hline Hazard ratio & 0.94 & & & & 0.95 & & \\
\hline$P$-value & & 0.71 & & & & 0.68 & \\
\hline Interaction $P$-value & & & & 0.98 & & & \\
\hline \multicolumn{8}{|l|}{ Objective response } \\
\hline Response rate (\%) & 40 & & 39 & & 27 & & 26 \\
\hline Odds ratio & $\mathrm{I} .0 \mathrm{I}$ & & & & 1.06 & & \\
\hline$P$-value & & 0.98 & & & & 0.84 & \\
\hline \multirow[t]{4}{*}{ Interaction $P$-value } & & & & 0.91 & & & \\
\hline & \multicolumn{7}{|l|}{ SQUIRE $^{25}$} \\
\hline & \multicolumn{3}{|l|}{ H-score high } & & \multicolumn{3}{|l|}{ H-score low } \\
\hline & CDDP-GEM-NEC & & CDDP-GEM & & CDDP-GEM-NEC & & CDDP-GEM \\
\hline Patients, $\mathrm{n}$ & 191 & & 183 & & 295 & & 313 \\
\hline \multicolumn{8}{|l|}{ Overall survival } \\
\hline Median (months) & 12.0 & & 9.7 & & 11.1 & & 10.9 \\
\hline Hazard ratio & 0.75 & & & & 0.90 & & \\
\hline$P$-value & & 0.01 & & & & 0.23 & \\
\hline Interaction $P$-value & & & & 0.24 & & & \\
\hline \multicolumn{8}{|c|}{ Progression-free survival } \\
\hline Median (months) & 5.7 & & 5.5 & & 5.7 & & 5.5 \\
\hline Hazard ratio & 0.88 & & & & 0.83 & & \\
\hline$P$-value & & 0.28 & & & & 0.04 & \\
\hline Interaction $P$-value & & & & 0.68 & & & \\
\hline \multicolumn{8}{|l|}{ Objective response } \\
\hline Response rate (\%) & 29 & & 30 & & 34 & & 28 \\
\hline Odds ratio & 0.97 & & & & 1.27 & & \\
\hline$P$-value & & 0.88 & & & & 0.17 & \\
\hline Interaction $P$-value & & & & 0.34 & & & \\
\hline
\end{tabular}

Abbreviations: CDDP, cisplatin; GEM, gemcitabine; NEC, necitumumab; PEM, pemetrexed.

every 21 days alone (548 patients) or in combination with necitumumab $800 \mathrm{mg}$ on days 1 and 8 (545 patients); similar to INSPIRE, this trial was open label and, after six cycles of chemotherapy, patients in the experimental arm with controlled or responding disease were allowed to receive maintenance with necitumumab at the same dose. In this study, the safety assessment showed that skin toxicity was the most common adverse event associated with necitumumab (any grade: $79 \%$, grade $\geq 3 \%: 8 \%$ ), while it was less frequent in the control arm (any grade: $12 \%$, grade $\geq 3 \%$ : $<1 \%$ ); similarly, the incidence of venous thromboembolic events was higher in the arm containing chemotherapy and necitumumab (any grade: $9 \%$, grade $\geq 3 \%: 5 \%$ ) than in the arm containing chemotherapy alone (any grade: $5 \%$, grade $\geq 3 \%$ : $3 \%$ ), although the rate of fatal thromboembolic events (including both arterial and venous events) was low and not different between the two arms. In the efficacy analysis, the experimental arm achieved a significant advantage in terms of PFS (5.7 vs 5.5 months; $\mathrm{HR}=0.85 ; 95 \% \mathrm{CI}=0.74-0.98 ; P=0.02$ ) and $\mathrm{OS}$ ( 11.5 vs 9.9 months; $\mathrm{HR}=0.84 ; 95 \% \mathrm{CI}=0.74-0.96$; $P=0.01$ ); RR was not significantly different between the experimental and the control arm (31\% vs $29 \% ; P=0.40)$, while DCR favored the arm containing necitumumab ( $82 \%$ vs $77 \% ; P=0.043$ ). The analysis based on EGFR H-score (with the cut-off between low and high put at 200, as in INSPIRE) did not demonstrate a different benefit within 
H-score subpopulations (H-score high: 38\%; H-score low: $62 \%$ ), as the survival advantage with necitumumab was confirmed in both subgroups and no significant difference was observed in each arm for $\mathrm{H}$-score high vs $\mathrm{H}$-score low, both in terms of PFS (experimental arm: 5.7 vs 5.7 months; $\mathrm{HR}=1.03 ; 95 \% \mathrm{CI}=0.84-1.26 ; P=0.80 ;$ standard arm: $5.5 \mathrm{vs}$ 5.5 months; $\mathrm{HR}=0.96 ; 95 \% \mathrm{CI}=0.78-1.19 ; P=0.74)$ and $\mathrm{OS}$ (experimental arm: 12.0 vs 11.1 months; HR=0.94; 95\% $\mathrm{CI}=0.76-1.16 ; P=0.80$; standard arm: 9.7 vs 10.9 months; $\mathrm{HR}=1.12 ; 95 \% \mathrm{CI}=0.92-1.37 ; P=0.26)$. The authors reported that the HR for OS between the experimental and the standard arm in the EGFR H-score high population was 0.75 (95\% CI $=0.60-0.94)$, while the HR in the H-score low population was 0.90 (95\% $\mathrm{CI}=0.75-1.07)$; notably, the $\mathrm{HR}$ for PFS apparently followed a different trend, as it was 0.88 $(95 \% \mathrm{CI}=0.70-1.11)$ in the $\mathrm{H}$-score high and $0.83(95 \%$ $\mathrm{CI}=0.69-0.99)$ in the $\mathrm{H}$-score low populations. However, the interaction $P$-value was not significant for OS and PFS (0.24 and 0.68 , respectively). ${ }^{25}$

The SQUIRE trial was subject to several further analyses, which added information about specific subgroups, quality of life, and potential predictive factors. In a subgroup analysis evaluating EGFR expressing patients (regardless of $\mathrm{H}$-score) and patients with completely undetectable EGFR protein, most evaluable patients (95.2\%) had some expression of EGFR and their characteristics were well balanced between the treatment arms, while only $4.8 \%$ were totally negative for EGFR; in the EGFR-positive population, the addition of necitumumab resulted in a significant improvement in terms of PFS (HR $=0.84 ; 95 \% \mathrm{CI}=0.72-0.97 ; P=0.018)$ and $\mathrm{OS}(\mathrm{HR}=0.79 ; 95 \% \mathrm{CI}=0.69-0.92 ; P=0.002)$ over the control arm, while in the subgroup of patients who were EGFR negative, no advantage in terms of PFS (HR $=1.33$; 95\% CI $=0.65-2.70 ; P=0.428)$ or $\mathrm{OS}(\mathrm{HR}=1.52 ; 95 \%$ $\mathrm{CI}=0.74-3.12 ; P=0.428)$ was observed with the addition of necitumumab to chemotherapy. ${ }^{27}$

Although SQUIRE was not designed to assess the safety and the effectiveness of continuing necitumumab after completing the combination, a post hoc analysis was conducted on those patients who were alive and progression-free after completion of chemotherapy; the HRs for OS and PFS in the post-induction period were 0.85 and 0.86 , respectively, consistent with the overall SQUIRE data. ${ }^{28}$

Key subgroup analyses, including Eastern Cooperative Oncology Group performance status, sex, ethnicity, and age resulted in a generally consistent OS advantage in the experimental arm; this advantage was not confirmed in patients aged $\geq 70$ years $(H R \approx 1.0)$, although the drug exposure for this subpopulation was reportedly reduced. ${ }^{29-31}$ Furthermore, the efficacy and the safety observed in a subpopulation of 84 East Asian patients from the SQUIRE trial (43 enrolled in the experimental arm and 41 enrolled in the control arm) were reported as generally consistent with data from nonEast Asian patients. ${ }^{32}$

With regard to quality of life, an exploratory post hoc analysis of the lung cancer symptom scale did not show significant differences in terms of time to symptom deterioration or in terms of required supportive care between the experimental and the standard arm, while the baseline maximum severity score, defined as the worst (highest) score of any individual lung cancer symptom scale item at baseline, was suggested to have some prognostic and predictive value with the limitations of this analysis; notably, the addition of necitumumab resulted in better HR values in the subgroups with higher maximum severity score. ${ }^{33}$

Finally, a retrospective analysis evaluated the value of EGFR gene copy number assessed with fluorescence in situ hybridization (FISH), as a predictor of outcome in 557 evaluable patients; although a positive FISH does not qualify as a predictor of outcome with statistical significance, a trend toward significance was observed for both PFS (interaction $P=0.057$ ) and $\mathrm{OS}$ (interaction $P=0.066$ ), and the addition of necitumumab in the FISH-positive population resulted in an HR equal to 0.71 for PFS ( $95 \% \mathrm{CI}=0.52-0.97)$ and 0.70 for OS $(95 \%$ CI $=0.52-0.96) .{ }^{34}$

\section{Safety profile}

The different trials involving necitumumab allowed the investigators to collect meaningful data about its safety profile and the drug-related adverse events. The main necitumumabrelated adverse events observed at the approved dose of $800 \mathrm{mg}$ in combination with chemotherapy were collected from INSPIRE and SQUIRE trials ${ }^{24,25}$ and are reported in Table 3. Notably, since the administration of necitumumab to nonprogressing patients was continued after completion of induction chemotherapy, the safety monitoring period was longer on the experimental arm, possibly introducing a bias favoring the control arm.

As previously reported, since EGFR is the main target of necitumumab, the most frequent toxicity involves skin reactions. This toxicity is explained by the presence of EGFR on a number of epidermal cells, including keratinocytes and sebocytes, ${ }^{35}$ and becomes manifest in the form of dry skin, acne, dermatitis, skin ulcer, or pruritus; most of these adverse events, however, were mild or moderate (grade 1-2). This toxicity is consistent with the reported 
Table 3 Relevant drug-related toxicities from trials involving necitumumab in combination with platinum-based chemotherapy in advanced NSCLC, based on published data from INSPIRE ${ }^{24}$ and SQUIRE ${ }^{25}$

\begin{tabular}{|c|c|c|c|c|}
\hline \multirow[t]{2}{*}{ Adverse event } & \multicolumn{2}{|c|}{$\begin{array}{l}\text { Necitumumab plus cisplatin } \\
\text { and pemetrexed }\end{array}$} & \multicolumn{2}{|c|}{$\begin{array}{l}\text { Necitumumab plus cisplatin } \\
\text { and gemcitabine }\end{array}$} \\
\hline & $\%$ any grade & $\%$ grade $\geq 3$ & $\%$ any grade & $\%$ grade $\geq 3$ \\
\hline Skin disorders & 78 & 15 & 79 & 8 \\
\hline Rash & 41 & 8 & 44 & 4 \\
\hline Dermatitis acneiform & 14 & 3 & 15 & I \\
\hline Acne & 4 & $<1$ & 9 & $<1$ \\
\hline Pruritus & 10 & $<1$ & 7 & $<1$ \\
\hline Skin ulcer & $<1$ & $<1$ & $<1$ & $<1$ \\
\hline Fatigue & 56 & II & 42 & 7 \\
\hline Hypomagnesemia & 27 & 8 & 31 & 9 \\
\hline Eye disorders & 16 & 0 & 7 & $<1$ \\
\hline Venous thromboembolic events & 13 & 8 & 9 & 5 \\
\hline Cardio-respiratory arrest/sudden death & 0 & $<1$ & 0 & 3 \\
\hline
\end{tabular}

Abbreviation: NSCLC, non-small cell lung cancer.

profiles for other drugs belonging to the same class and should be managed in the same way. ${ }^{36}$ Notably, a correlation between occurrence of skin rash during the first two cycles and improved OS was suggested on the basis of an analysis of SQUIRE, ${ }^{37}$ which was consistent with similar observations reported with cetuximab. ${ }^{4}$ When taking into account blood examinations and laboratory values, the addition of necitumumab to chemotherapy did not result in an increased rate of anemia, leukopenia, or thrombocytopenia, while the most significant laboratory-related alteration was a reduction in magnesium levels; such toxicity is related to the presence of EGFR on cells forming distal and collecting tubules in the kidneys, which are normally involved in the regain of magnesium, and is further worsened by the renal toxicity associated with cisplatin (known as a magnesiumdepleting agent). Although the actual clinical relevance of this toxicity has yet to be defined properly, ${ }^{38}$ it is possible that electrolytes disorders might partially be correlated with cardiac dysfunctions; furthermore, a cardiac arrest/sudden death ratio of $3 \%$ (compared to $0.6 \%$ in the control arm) was reported in SQUIRE, although most of these patients were affected by cardiologic comorbidities; however, it should be taken into account that cisplatin-based regimens are commonly associated with hypomagnesemia. ${ }^{26,39}$ Some adverse events at least partially correlated with the addition of necitumumab to chemotherapy were observed with a different frequency according to the employed regimen and were generally more relevant to the cisplatin-pemetrexed combination. The most concerning toxicity was represented by venous thromboembolic events, a significant proportion of which were severe $(5 \%-8 \%)$, while the incidence of arterial thromboembolic events did not seem to be increased by the use of necitumumab or other EGFR mABs. While it should be noted that venous thromboembolism is common in lung cancer patients, and especially in those patients receiving chemotherapy, this toxicity apparently characterizes the whole drug class (while it is not shared with EGFR tyrosine kinase inhibitors); however, its physiopathological mechanisms are still unclear, although they might be correlated to an indirect disruption of the vascular endothelial growth factor signaling. ${ }^{40}$ Fatigue was another commonly observed adverse event, and, although it is a typical side effect of combination chemotherapy, the authors of the INSPIRE trial reported that its frequency was increased in the combination of cisplatin-pemetrexed-necitumumab compared to cisplatin-pemetrexed, while no significant difference was reported in SQUIRE. Another category of adverse events at least partially associated with the association of necitumumab and chemotherapy with cisplatin-pemetrexed was represented by eye disorders, which included events such as dryness or conjunctivitis. Finally, the allergy-related adverse events were not significantly higher in the experimental arms of INSPIRE and SQUIRE; this difference between necitumumab and cetuximab, which is characterized by a high prevalence of hypersensitivity reactions, might be explained by the fully human structure of the former and the chimeric structure of the latter. ${ }^{41}$

\section{Regulatory affairs}

Based on the data from SQUIRE, in November 2015, necitumumab was approved by the US Food and Drug Administration for the first-line treatment of advanced SCC in combination with cisplatin and gemcitabine. ${ }^{42}$ In December 2015, a positive opinion from the Committee for Medicinal 
Products for Human Use of the European Medicines Agency (EMA) ultimately resulted in the authorization for the use of necitumumab in combination with cisplatin and gemcitabine as the first-line treatment for adult patients affected by EGFR-expressing advanced squamous NSCLC in February 2016. ${ }^{43,44}$ While testing for EGFR protein expression is not necessary in order to prescribe necitumumab according to US Food and Drug Administration, the specific request from EMA is connected to the subgroup analysis reporting that, as previously described, a positive EGFR protein expression was identified in specimens from nearly all the patients evaluable for IHC in the SQUIRE trial and that the smaller proportion of patients with undetectable EGFR expression did not achieve improved outcomes with the addition of necitumumab.

\section{Discussion}

The most relevant advances in the treatment of advanced NSCLC over the past decade involved almost exclusively non-squamous histology, mostly thanks to the exploitation of oncogenic drivers. Necitumumab represents quite a unique model in the lung cancer landscape, as its addition to standard chemotherapy resulted in a significant survival advantage for patients with SCC, making it the first targeted agent to be approved in combination with first-line chemotherapy for squamous histology. In the Phase III trials involving the addition of necitumumab to chemotherapy, the benefit observed in SCC (as well as its lack of in non-squamous histology) was generally consistent among subpopulations selected on the basis of clinical features and EGFR expression measured as $\mathrm{H}$-score, although no survival benefit was demonstrated in a limited population of patients with SCC lacking EGFR expression. Notably, the lack of benefit in this population induced EMA to limit the availability of necitumumab to EGFR-positive patients in Europe, although this decision was based on a relatively small subpopulation (4.8\%) compared to the global SQUIRE population; further trials and analyses might eventually add valuable information to this specific setting.

One possible limitation to the use of necitumumab lies in the current lack of prospective data on reliable predictive biomarkers that are able to improve patient selection; in fact, the addition of necitumumab to standard chemotherapy is associated with increased toxicity, albeit in line with the drug class. Additionally, as the cost-benefit value of new antineoplastic agents is a major cause of concern at the current time, ${ }^{45}$ being able to select the patients who are going to benefit the most from the addition of necitumumab might actually improve its cost-benefit ratio and ultimately increase its value. Notably, while a retrospective analysis of SQUIRE reported improved HR for OS and PFS in patients with increased EGFR gene copy number determined by FISH, the retrospective nature of this evaluation does not allow to draw definitive conclusions, but at the same time might generate additional working hypotheses, which might be the basis of prospective studies designed to explore the role of FISH in necitumumab-based regimens.

Finally, further studies involving necitumumab are being planned or are currently ongoing; one of these studies is of particular relevance, as it aims to explore the possible role of the drug in association with osimertinib (a recently approved EGFR inhibitor specifically designed to address the T790M mutation), actually acting as a dual blockade on the receptor; ${ }^{46}$ despite being a Phase I trial, this study might open some additional perspectives for further development of this agent. Other trials are currently exploring the effect of combining necitumumab with other systemic treatments, such as alternative chemotherapy regimens, ${ }^{47}$ investigational targeted therapies, ${ }^{48,49}$ or immune check-point inhibitors. ${ }^{50}$

In summary, necitumumab is an interesting addition to the therapeutic options available to medical oncologists and patients with advanced SCC and might have a role in filling the current therapeutic gap between squamous and nonsquamous lung cancer; additionally, further studies addressing selected patient populations might potentially extend the role of this drug in the treatment of advanced NSCLC, improve its risk-benefit ratio, and increase its cost-effectiveness.

\section{Acknowledgment}

The authors wish to acknowledge Dr Javad Shahidi (Eli Lilly and Company) for providing writing assistance.

\section{Disclosure}

Dr Fred R Hirsch has received compensation for advisory boards and research fundings from Eli Lilly and Company. Dr Carlo Genova reports no relevant conflicts of interest in this work.

\section{References}

1. Howlader N, Noone AM, Krapcho M, et al. SEER Cancer Statistics Review, 1975-2012, National Cancer Institute. Bethesda, MD. Available from: http://seer.cancer.gov/csr/1975_2012/, based on November 2014 SEER data submission, posted to the SEER website. April 2015.

2. Masters GA, Temin S, Azzoli CG, et al. Systemic therapy for stage IV non-small-cell lung cancer: American Society of Clinical Oncology Clinical Practice Guideline update. J Clin Oncol. 2015;33(30) 3488-3515. 
3. Hirsch FR, Varella-Garcia M, Bunn PA Jr, et al. Epidermal growth factor receptor in non-small-cell lung carcinomas: correlation between gene copy number and protein expression and impact on prognosis. J Clin Oncol. 2003;21(20):3798-3807.

4. Pirker R, Pereira JR, Szczesna A, et al. Cetuximab plus chemotherapy in patients with advanced non-small-cell lung cancer (FLEX): an openlabel randomised phase III trial. Lancet. 2009;373(9674):1525-1531.

5. Pirker R, Pereira JR, von Pawel J, et al. EGFR expression as a predictor of survival for first-line chemotherapy plus cetuximab in patients with advanced non-small-cell lung cancer: analysis of data from the phase 3 FLEX study. Lancet Oncol. 2012;13(1):33-42.

6. Tay RY, Wong R, Hawkes EA. Treatment of metastatic colorectal cancer: focus on panitumumab. Cancer Manag Res. 2015;7:189-198.

7. Goffin JR, Zbuk K. Epidermal growth factor receptor: pathway, therapies, and pipeline. Clin Ther. 2013;35(9):1282-1303.

8. Schmitz KR, Bagchi A, Roovers RC, van Bergen en Henegouwen PM, Ferguson KM. Structural evaluation of EGFR inhibition mechanisms for nanobodies/VHH domains. Structure. 2013;21(7):1214-1224.

9. Li S, Kussie P, Ferguson KM. Structural basis for EGF receptor inhibition by the therapeutic antibody IMC-11F8. Structure. 2008;16(2): 216-227.

10. Dienstmann R1, Felip E. Necitumumab in the treatment of advanced non-small cell lung cancer: translation from preclinical to clinical development. Expert Opin Biol Ther. 2011;11(9):1223-1231.

11. Bakema JE, van Egmond M. Fc receptor-dependent mechanisms of monoclonal antibody therapy of cancer. Curr Top Microbiol Immunol. 2014;382:373-392.

12. Keizer RJ, Huitema AD, Schellens JH, Beijnen JH. Clinical pharmacokinetics of therapeutic monoclonal antibodies. Clin Pharmacokinet. 2010;49(8):493-507.

13. Kuenen B, Witteveen PO, Ruijter R, et al. A phase I pharmacologic study of necitumumab (IMC-11F8), a fully human IgG1 monoclonal antibody directed against EGFR in patients with advanced solid malignancies. Clin Cancer Res. 2010;16(6):1915-1923.

14. Liu M, Zhang H, Jimenez X, et al. Identification and characterization of a fully human antibody directed against epidermal growth factor receptor for cancer therapy. Proc Am Assoc Cancer Res. 2004;45: Abstract 706.

15. Saxena B, Sundaram ST, Walton W, et al. Differentiation between the EGFR antibodies necitumumab, cetuximab, and panitumumab: In vitro biological and binding activities. J Clin Oncol. 2011;29 Suppl: Abstract e13030.

16. Topper MB, Tonra JR, Pytowski B, Eastman SW. Differentiation between the EGFR antibodies necitumumab, cetuximab, and panitumumab: Antibody internalization and EGFR degradation. J Clin Oncol. 2011;29 Suppl: Abstract e13022.

17. Patel D, Saxena B, Zhou Q, et al. Differential induction of antibody-dependent cellular cytotoxicity (ADCC) against human EGFR-expressing NSCLC cell lines by necitumumab, cetuximab, and panitumumab. J Clin Oncol. 2011;29 Suppl: Abstract e21075.

18. Yu D, Wuertz J, Taqui A, et al. Differentiation between the EGFR antibodies necitumumab (Neci), cetuximab (Cetux), and panitumumab (Pan): Glycosylation and IgE reactivity. J Clin Oncol. 2011;29 Suppl: Abstract e13023.

19. Prewett M, Tonra JR, Rajiv B, Hooper AT. Antitumor activity of a novel, human anti-epidermal growth factor receptor (EGFR) monoclonal antibody (IMC-11F8) in human tumor xenograft models. Proc Am Assoc Cancer Res. 2004;45: Abstract 5353.

20. Samakoglu S, Deevi DS, Li H, et al. Preclinical rationale for combining an EGFR antibody with cisplatin/gemcitabine for the treatment of NSCLC. Cancer Genomics Proteomics. 2012;9(2):77-92.

21. Nokihara H, Yamamoto N, Tamura Y, et al. A phase 1 study of necitumumab (anti-EGFR monoclonal antibody) in Japanese patients with advanced solid tumors. Ann Oncol. 2014;25 Suppl 5:v44-v74.

22. Corral J, Ponce S, Aerts JG, et al. A phase II study of gemcitabinecisplatin plus necitumumab for stage IV Sq-NSCLC. J Thorac Oncol. 2015;10(9):S153.
23. Spigel D, Luft A, Ramlau R, et al. A randomized, multicenter, openlabel, phase 2 study of paclitaxel-carboplatin (PC) chemotherapy plus necitumumab (IMC-11F8/LY3012211) versus PC alone in the first-line treatment of patients (pts) with stage IV squamous non-small cell lung cancer (sq-NSCLC). Presented at: the AACR-NCI-EORTC International Conference of Molecular Targets and Cancer Therapeutics; 5-9 November 2015; Boston, MA (Abstract: C122).

24. Paz-Ares L, Mezger J, Ciuleanu TE, et al. Necitumumab plus pemetrexed and cisplatin as first-line therapy in patients with stage IV non-squamous non-small-cell lung cancer (INSPIRE): an open-label, randomised, controlled phase 3 study. Lancet Oncol. 2015;16(3):328-337.

25. Thatcher N, Hirsch FR, Luft AV, et al. Necitumumab plus gemcitabine and cisplatin versus gemcitabine and cisplatin alone as first-line therapy in patients with stage IV squamous non-small-cell lung cancer (SQUIRE): an open-label, randomised, controlled phase 3 trial. Lancet Oncol. 2015;16(7):763-774.

26. FDA Briefing Document Oncologic Drugs Advisory Committee Meeting (BLA 125547/0 - Necitumumab); 2015. Available from: http://www.fda. gov/downloads/AdvisoryCommittees/CommitteesMeetingMaterials/ Drugs/OncologicDrugsAdvisoryCommittee/UCM453752.pdf. Accessed on May 20, 2016.

27. Paz-Ares L, Socinski MA, Shahidi J, et al. Subgroup analyses of patients with epidermal growth factor receptor (EGFR)-expressing tumors in SQUIRE: a randomized, multicenter, open-label, phase III study of gemcitabine-cisplatin (GC) plus necitumumab $(\mathrm{N})$ versus $\mathrm{GC}$ alone in the first-line treatment of patients (pts) with stage IV squamous nonsmall cell lung cancer (sq-NSCLC). Presented at: the 6th European Lung Cancer Conference (ELCC); 13-16 April 2016; Geneva, Switzerland (1320_PR).

28. Ciuleanu TE, Socinski MA, Obasaju CK, et al. Efficacy and safety of necitumumab continuation therapy in phase 3 SQUIRE study. $J$ Thorac Oncol. 2015;10(9):S235-S236.

29. Socinski MA, Luft A, Szczesna A, et al. Subgroup analyses by performance status (PS) in the phase III SQUIRE study: first-line necitumumab $(\mathrm{N})$ plus gemcitabine-cisplatin (GC) vs GC in squamous non-small cell lung cancer (NSCLC). J Clin Oncol. 2015;33 Suppl: Abstract e1902.

30. Socinski MA, Paz-Ares L, Luft A, et al. SQUIRE: A randomized, multicenter, open-label, phase III study of gemcitabine-cisplatin (GC) chemotherapy plus necitumumab (IMC-11F8/LY3012211) vs GC alone in the first-line treatment of patients (pts) with stage IV squamous non-small cell lung cancer (sq-NSCLC): update on key subgroups. Ann Oncol. 2014;25 Suppl 4: Abstract 1232P.

31. Thatcher N, Ciuleanu T, Ramlau R, et al. Subgroup analysis of elderly patients in SQUIRE: A randomized, multicenter, open-label, phase III study of necitumumab (N) plus gemcitabine-cisplatin (GC) chemotherapy versus GC alone in first-line treatment of patients (pts) with stage IV squamous non-small cell lung cancer (sq-NSCLC). Ann Oncol. 2015;26 Suppl 1: Abstract 103PD.

32. Park K, Cho EK, Bello M, et al. Efficacy and safety of necitumumab (neci) in East Asian (EA) patients (pts) with stage IV squamous nonsmall-cell lung cancer (NSCLC): a subanalysis of the SQUIRE trial. Ann Oncol. 2015;26 Suppl 1: Abstract 436P.

33. Reck M, Socinski MA, Luft A, et al. The effect of necitumumab in combination with gemcitabine plus cisplatin on tolerability and on quality of life: results from the phase III SQUIRE trial. J Thorac Oncol. 2016; 11(6):808-818.

34. Paz-Ares L, Socinski MA, Shahidi J, et al. Correlation of EGFRexpression with safety and efficacy outcomes in SQUIRE: a randomized, multicenter, open-label, phase III study of gemcitabine-cisplatin plus necitumumab versus gemcitabine-cisplatin alone in the first-line treatment of patients with stage IV squamous non-small cell lung cancer. Ann Oncol. 2016;27(8):1573-1579.

35. Baas JM, Krens LL, Guchelaar HJ, et al. Recommendations on management of EGFR inhibitor-induced skin toxicity: a systematic review. Cancer Treat Rev. 2012;38(5):505-514. 
36. Belum VR1, Cercek A, Sanz-Motilva V, Lacouture ME. Dermatologic adverse events to targeted therapies in lower GI cancers: clinical presentation and management. Curr Treat Options Oncol. 2013;14(3): 389-404.

37. Bonomi P, Peterson P, Socinski M, et al. Rash as a marker for the efficacy of necitumumab in the SQUIRE study. J Thorac Oncol. 2015; 10(9) suppl: Abstract 1-62.

38. Petrelli F, Borgonovo K, Cabiddu M, Ghilardi M, Barni S. Risk of anti-EGFR monoclonal antibody-related hypomagnesemia: systematic review and pooled analysis of randomized studies. Expert Opin Drug Saf. 2012;11 Suppl 1:S9-S19.

39. Saif MW. Management of hypomagnesemia in cancer patients receiving chemotherapy. J Support Oncol. 2008;6(5):243-248.

40. Petrelli F, Cabiddu M, Borgonovo K, Barni S. Risk of venous and arterial thromboembolic events associated with anti-EGFR agents: a meta-analysis of randomized clinical trials. Ann Oncol. 2012;23(7): 1672-1679.

41. Chung CH, Mirakhur B, Chan E, et al. Cetuximab-induced anaphylaxis and IgE specific for galactose-alpha-1,3-galactose. N Engl J Med. 2008; 358(11):1109-1117.

42. Food and Drug Administration news release: FDA approves Portrazza to treat advanced squamous non-small cell lung cancer; 2015. Available from: http://www.fda.gov/NewsEvents/Newsroom/ PressAnnouncements/ucm474131.htm. Accessed March 3, 2016.

43. European Medicines Agency: summary of opinion (initial authorization) of the Committee for Medicinal Products for Human Use (CHMP) on Portrazza; 2015. Available from: http://www.ema.europa.eu/docs/en GB/document_library/Summary_of_opinion_-_Initial_authorisation/ human/003886/WC500199044.pdf. Accessed March 3, 2016.

44. European Medicines Agency: product information. Necitumumab. Available from: http://www.ema.europa.eu/docs/en_GB/document_ library/EPAR_-_Product_Information/human/003886/WC500202694. pdf. Accessed March 31, 2016.
45. Goldstein DA, Chen Q, Ayer T, et al. Necitumumab in metastatic squamous cell lung cancer: establishing a value-based Cost. JAMA Oncol. 2015;1(9):1293-1300.

46. National Cancer Institute. EGFR Inhibitor AZD9291 and Necitumumab in Treating Patients With EGFR-Positive Stage IV or Recurrent Non-small Cell Lung Cancer Who Have Progressed on a Previous EGFR Tyrosine Kinase Inhibitor. Available from: https://www.clinicaltrials.gov/ct2/ show/NCT02496663?term=necitumumab\&rank=3. NLM identifier: NCT02496663. Accessed September 30, 2015.

47. Eli Lilly and Company. A Study of Nab-Paclitaxel and Carboplatin Plus Necitumumab (LY3012211) in Participants With Stage IV Squamous NSCLC. Available from: https://www.clinicaltrials.gov/ct2/show/NCT0 2392507 ?term=necitumumab\&rank=7. NLM identifier: NCT02392507. Accessed September 30, 2015.

48. Eli Lilly and Company. A Study of Necitumumab and Abemaciclib in Participants With Non-Small Cell Lung Cancer (NSCLC). Available from: https:/www.clinicaltrials.gov/ct2/show/NCT02411591?term=ne citumumab+lung\&rank=7. NLM identifier: NCT02411591. Accessed May 20, 2016.

49. Eli Lilly and Company. A Study of LY3023414 and Necitumumab in Squamous Lung Cancer. Available from: https://www.clinicaltrials. gov/ct2/show/NCT02443337?term=necitumumab+lung\&rank=5. NLM identifier: NCT02443337. Accessed May 20, 2016.

50. Eli Lilly and Company. A Study of the Combination of Necitumumab (LY3012211) and Pembrolizumab (MK3475) in Participants With NSCLC. Available from: https://www.clinicaltrials.gov/ct2/show/N CT02451930?term=necitumumab+lung\&rank=10. NLM identifier: NCT02451930. Accessed May 20, 2016
OncoTargets and Therapy

\section{Publish your work in this journal}

OncoTargets and Therapy is an international, peer-reviewed, open access journal focusing on the pathological basis of all cancers, potential targets for therapy and treatment protocols employed to improve the management of cancer patients. The journal also focuses on the impact of management programs and new therapeutic agents and protocols on

\section{Dovepress}

patient perspectives such as quality of life, adherence and satisfaction. The manuscript management system is completely online and includes a very quick and fair peer-review system, which is all easy to use. Visit http://www.dovepress.com/testimonials.php to read real quotes from published authors. 